\title{
Research priorities in suicide prevention: A qualitative study of stakeholders' views
}

\author{
Sara Niner, ${ }^{1}$ Jane Pirkis, ${ }^{1}$ Karolina Krysinska, ${ }^{2}$ Jo Robinson, ${ }^{3}$ Michael Dudley, ${ }^{4,5}$ \\ Emily Schindeler, ${ }^{5,6}$ Diego De Leo ${ }^{2}$ and Deborah Warr ${ }^{7}$
}

1. Centre for Health Policy, Programs and Economics, School of Population Health, The University of Melbourne, Melbourne, Australia

2. Australian Institute for Suicide Research and Prevention, Griffith University, Brisbane, Australia

3. ORYGEN Research Centre, The University of Melbourne, Melbourne, Australia

4. School of Psychiatry, The University of New South Wales, Sydney, Australia

5. Suicide Prevention Australia, Sydney, Australia

6. S \& S Consultants, Brisbane, Australia

7. McCaughey Centre, School of Population Health, The University of Melbourne, Melbourne, Australia

\begin{abstract}
The current study sought to inform priority setting in Australian suicide prevention research, by seeking stakeholders' views on where future priorities might lie. Three group interviews were conducted with a total of 28 participants. Group interview participants stressed that priority should be given to evaluating the efficacy of specific interventions and examining the response of the health and community service systems. They felt that the epidemiological profile of suicidal individuals had been explored, at least with respect to rates and individual-level risk factors, and that the above evaluative activities should focus on groups identified as having particularly high levels of risk. Most saw limited value in continuing to explore individual-level risk factors ad infinitum, and felt that the time had come to move on to considering wider societal influences on suicide and individual-level protective factors. Many felt that evaluation efforts should employ mixed methods, should be multidisciplinary and should be relevant to the Australian context. They also argued that there was scope for increasing the utility of research findings by communicating them in a manner that would enable them to be utilised by policy-makers, planners and practitioners. Several called for a more cohesive framework for suicide prevention that could guide suicide prevention research. The current study provides some guidance with respect to the direction Australia's suicide prevention research agenda should take. A prioritydriven approach to suicide prevention research will ensure that the research endeavour provides the most useful information for those whose day-to-day work involves trying to prevent suicide.
\end{abstract}

\section{Keywords}

suicide, suicide prevention, national strategies, evaluation, qualitative

\section{Background}

Since 1999, Australia's suicide prevention efforts have been guided by a co-ordinated, overarching framework known as the LIFE Framework (Australian Government Department of Health and Ageing, 2008a) which is operationalised by the Australian Government via the National Suicide Prevention Strategy. The LIFE Framework has placed strong emphasis on seeking the best available evidence for its preventive activities. There is a recognition among both the Australian and international suicide prevention communities,

Contact: $\quad$ Associate Professor Jane Pirkis, Centre for Health Policy, Programs and Economics, School of Population Health, The University of Melbourne, Victoria, Australia j.pirkis@unimelb.edu.au

Citation: $\quad$ Niner, S., Pirkis, J., Krysinska, K., Robinson, J., Dudley, M., Schindeler, E., De Leo, D., \& Warr, D. (2009). Research priorities in suicide prevention: A qualitative study of stakeholders' views. Australian e-Journal for the Advancement of Mental Health, 8(1), www.auseinet.com/journal/vol8iss1/niner.pdf

Published by: Australian Network for Promotion, Prevention and Early Intervention for Mental Health (Auseinet) www.auseinet.com/journal

Received 13 October 2008; Revised 7 April 2009; Accepted 7 April 2009 
however, that this evidence-base is as yet suboptimal, and that our knowledge of what works and what does not work in suicide prevention is far from perfect (Australian Government Department of Health and Ageing, 2008b; Linehan, 2008; Mann, Apter, Bertolote et al., 2005).

For this reason, the National Suicide Prevention Strategy is explicitly fostering the development of a national suicide prevention research agenda with a view to filling some of the gaps in this knowledge base. We were funded by the Australian Government Department of Health and Ageing to conduct the current project as the first step in developing such an agenda, and to encourage further research in the field. The project sought to inform priority setting in suicide prevention research, by empirically examining existing priorities and by seeking stakeholders' views on where future priorities might lie.

The project involved two studies. Study 1 empirically examined existing priorities (via a review of the published literature and a review of funded grants) and sought stakeholders' views on future priorities via a questionnaire. Together, Study 1's components generated quantitative data which permitted direct, relatively high-level comparisons between the status quo and stakeholders' views, and the findings have been reported elsewhere (Robinson, Pirkis, Krysinska et al., 2008). Study 2 also sought stakeholders' views, but did so in a more in-depth manner, via a series of group interviews. It is Study 2 that is the focus of the current paper.

\section{Method}

We conducted three group interviews: one in Melbourne with seven participants, one in Sydney with 11 participants, and one in Brisbane with 10 participants. Participants were purposefully sampled using a snowballing technique that began with a core group of individuals identified from our own networks (Patton, 1990). This ultimately yielded key informants who conducted, used and/or were involved in the funding of suicide prevention research, and/or who had been affected by suicide. These key informants were asked to consider the priority given to suicide prevention research to date, and then to discuss whether this emphasis should change in the future. The discussion was guided by a moderator (Liamputtong Rice \& Ezzy, 1999), using the prompts listed in Box 1. The group interviews can perhaps best be regarded as consensus panels, which are groups gathered to come to some agreement regarding a particular issue. In this case, they comprised key informants from the suicide prevention field and sought consensus or normative reaction regarding priorities in suicide prevention research via relatively narrow prompts (Coreil, 1995).

The discussion at each group interview was recorded on audiotape, and extensive notes were taken by a note-taker (who was not the moderator) (Liamputtong Rice \& Ezzy, 1999). Time and resource constraints prevented us from transcribing the full record of discussion, so the analysis relied primarily on the notes and the taped record. Having said this, parts of the recordings were fully transcribed in instances where the notes were unclear, where the discussion involved nuances that were not adequately reflected in the notes, or where the points being made were of particular relevance to a topic of interest.

A content analysis of the group interviews was conducted by one member of the research team, with recourse to the other team members who attended each group interview for any points of clarification and confirmation of interpretation. The content analysis required 'immersion' in the data via repeated listening to the tapes and reading of the notes (and transcripts, where applicable) (Streubert \& Carpenter, 1999). More specifically, the content analysis involved ident-

Box 1: Prompts used in group interviews

a. I'd like to start by asking you to think about our current level of knowledge about suicide prevention. What do we know?

b. What don't we know?

c. Thinking now about Australian suicide prevention research, what kind of research has taken priority in the last seven years (i.e., the duration of the National Suicide Prevention Strategy)?

d. Can you comment on the overall quality of Australian suicide prevention research?

e. Bearing in mind your responses to the earlier questions, what do you think should be the future priorities for suicide prevention research in Australia?

f. What factors do you think should influence future priorities for suicide prevention research in Australia? 
ifying key topics as they related to current and future priorities in suicide prevention. The topics became category labels, and interview segments or quotations were sorted into these categories. In instances where separate topics within a category emerged, the categories were further divided into sub-categories (Morse \& Field, 1995). Cross-coding of the notes (and transcripts) would have been desirable, but this was beyond the scope of the current study.

Because the aim was to seek consensus regarding priority areas for suicide prevention research, greatest emphasis was given to common responses within and across groups. This did not mean that the frequency of individual words was counted, nor that the number of stakeholders who made a particular point was quantified, because this is not regarded as appropriate practice in qualitative research (Morse \& Field, 1995). Instead, an effort was made to gauge whether a particular theme or sentiment recurred within a given category. Less common responses were noted and recorded, in an effort to explore the richness of variation in the data, but they were given less prominence when the findings were reported. This approach is consistent with the consensus panel method described by Coreil (1995) and was considered justified on the grounds that the study was designed to provide guidance to the Department of Health and Ageing regarding research priorities in suicide prevention.

\section{Results}

Although perspectives, experiences and views of group interview participants varied, several recurring themes emerged. These themes were consistent across the three group interviews and are summarised below.

\section{Evaluating the efficacy of interventions}

The most commonly raised issue across all three group interviews was the need for greater emphasis on the evaluation of interventions. Various participants observed that good epidemiological data exist on rates of suicide among particular population groups, and a reasonable amount of research has been conducted on risk and (to a lesser extent) protective factors, but that research into the efficacy of given interventions has been relatively neglected to date. Many felt that research resources should now be targeted towards evaluating the gamut of potential suicide prevention interventions. As one participant put it, 'Efficacy is a major issue.'

The majority of participants believed that suicide prevention interventions have been at best underevaluated and at worst unevaluated. Several commented that interventions and programs were designed using flimsy evidence, based on anecdotes and personal opinions. One participant summed this up in the following way:

'The system does have some theories about how it could prevent suicide, such as how to manage discharged patients from emergency departments, but none of these care models are well researched or evaluated ... If we look at assertive follow-up, case care etc., it's done with good intention but no-one knows the effectiveness of it.'

Several participants articulated the view that funding for intervention programs should explicitly include a budget for evaluation and knowledge development. One noted that this would require education on the evaluation process for service providers.

Some participants suggested specific intervention activities that required evaluation. These straddled the spectrum of interventions from mental health promotion (e.g., activities for strengthening resilience among young people), through primary and secondary prevention (e.g., early intervention and assertive follow-up with people presenting to mental health teams following suicide attempts), to treatment (e.g., cognitive behavioural therapy) and postvention (e.g., support groups for people who have attempted suicide and people who have been bereaved by suicide). In particular, there was consensus that interventions that seemed to show promise should be critically examined; such as, 'the intervention programs that have resulted in a turnaround of young male suicides since 1997.'

In a similar vein, several participants suggested that evaluation efforts should concentrate on people who have survived a suicide attempt. One participant, for example, said the following:

'Focus on those who have survived and what worked for them and what intervention helped at that time.'

Another made a similar but more specific comment, suggesting that:

'We need details of the therapeutic alliance. What is the variable for the person to survive?' 
Some participants argued that evaluative evidence is particularly lacking for interventions with specific subgroups. Indigenous communities are a case in point. One participant noted:

'From the Aboriginal perspective, there must be more work done in remote Aboriginal communities where suicide has been a big concern and issue. They have no consultants or counsellors. How did the community cope with suicide and what successful mechanisms did they put in place to recover from it? Have they been able to stop others from doing the same? We need to find this out and develop a model to transfer this knowledge to other situations. We need real statistics and information on causes and effects on the groups of interventions. We often only have assumptions.'

\section{Critically examining the response of the health and community service systems}

Beyond specific interventions, there was a view that critical examination of the service delivery system was needed. Many participants were critical of the current health system, claiming that it was ill-equipped to assess and manage people who presented with suicidal thoughts or behaviours. One participant cited GPs as an example, noting deficiencies in their ability to deal with suicidality:

'My GP couldn't tell after 11 years [of suicide attempts] ... I got treatment for myself ... he didn't notice the scars on my hands.'

Another participant spoke of difficulties for clinicians in the public and private mental health sectors:

'The quality of risk assessment in the public sector is ordinary/limited. Many are afraid to ask a question that they fear the answer to. People in the private sector are terrified to ask because what do they do with the answer? No beds are available anyway, so what do you do? ... Maybe the question is what has to be put into place for them to feel safer to ask the questions.'

Several participants felt that the solution to these problems lay in research into structured clinical assessment processes, clinical guidelines, risk management tools and professional judgement processes.

Mention was frequently made of the fact that many people who die by suicide receive services from different health and community services in the months, weeks and even days before death, often in relation to prior suicidal thoughts or behaviours. Participants noted that co-ordination between different sectors is sub-optimal, and that better communication between services might prevent some people 'falling through the gaps.' Several participants recommended that research to map these gaps could be useful in addressing them. Specifically, suggestions included research into the linkages between police and mental health services, between drug and alcohol services and mental health services, and between community services and clinical services.

Some participants stressed that a significant proportion of people who complete, attempt or consider suicide do not seek help, raising issues about the accessibility of services that warrants research attention. Some commented that it would be short-sighted to focus the entire research effort on those who do present to health and community services, making comments like:

'In all this we are still focusing on those people who present rather than on those people who don't present. The shocking question is: Why do people kill themselves? Is it factors outside the mental health system, way beyond clinical presentations to broader reasons? We need to have a systematic look at these issues.'

Several participants suggested that investigating the trajectory of suicidal behaviour via longitudinal methods may assist in this regard, because it might tease out where in the continuum people decide to act on suicidal ideation and why people choose to seek help or not to seek help. This in turn might provide clues about ways in which the health and community service systems can be optimally responsive.

\section{A focus on high risk groups}

Some participants felt that sufficient was known about high risk groups, at least in terms of their epidemiology. The majority, however, expressed the view that particular groups should still take precedence in terms of the suicide prevention research effort.

Men stood out as one such high risk group. Some participants felt that young male suicide has received significant attention, and that the reversal of their previously high suicide rate in Australia might mean that other groups should warrant research attention. Others argued, however, that young male suicide has not decreased among lower socio-economic groups, 
and that these groups should maintain research priority. Still others mentioned different subgroups of men, including older men, male prisoners and ex-prisoners, rural men, unemployed men, men in traditional male occupations and workplaces (e.g., builders, truck drivers), war veterans, recently separated men and men with relationship problems, and men with histories of sexual abuse. Several noted that many men do not seek help, and those who do are often not well served by services. One participant described this in the following way:

'Men don't access services because the services are disrespectful of men and the circumstances in which they might come to seek help.'

Older people were another commonly mentioned group. Many participants felt that older people had been marginalised and paid insufficient attention in suicide prevention research. For this reason, a number of participants felt that older people should become the new priority in suicide prevention research. Specific suggestions for the research focus among this group included identifying reasons for their high suicide rates, evaluating interventions that work for older people, and examining the blurring between euthanasia and suicide among the elderly.

A number of other specific groups were mentioned by at least one participant as requiring research attention. These included people detained in or recently released from immigration centres, people in Indigenous communities, members of the Stolen Generation (see Note), people who have experienced domestic violence, children in the juvenile justice system, children of recently-separated parents, people with drug and alcohol problems, people exhibiting suicidal behaviours (e.g., those presenting to emergency departments having deliberately self-harmed), people who have been bereaved by suicide and/or have a family history of suicide, people with mental health problems (particularly those who have been recently discharged from mental health services) and crisis line callers.

There was an acknowledgement that individual participants would 'lobby' for a focus on the high risk groups with whom they were most familiar, which led to discussion of how to develop more objective criteria for selecting particular groups of interest. One participant questioned how decisions are and should be made, asking others to consider why some groups have received more research attention than others. The participant questioned whether high group-specific suicide rates have made the difference, or whether other factors, such as perceived attractiveness or vulnerability, might have had an influence.

\section{Balancing individual risk factors with societal, environmental, cultural and political influences on suicide}

Most participants acknowledged that there are some clear individual-level risk factors for suicide, such as mental illness. However, several participants argued that there are also broader societal, environmental, cultural and political influences on suicide that to date have been under-researched. Some attributed this to the largely biomedical paradigm within which they perceived current suicide prevention research to be occurring.

Participants expressed this notion in different ways, using different examples. One mentioned suicide by those in or recently released from detention centres, suggesting that the political situation would need to be taken into account in researching appropriate and effective suicide prevention interventions for this group. Others talked about job insecurity, unemployment, rurality and low socio-economic status, recommending that anthropological and sociological issues must be addressed in understanding these factors and their impact on suicide. Still others mentioned cultural factors, suggesting, for example, that more research attention needs to be devoted to stigma and how this may impact on suicidality. One participant summed up the need to examine the influence that these factors can have on human lives in the following way:

'[We must] look at people's quality of life and the telltale signs [of despair].'

\section{Consideration of protective factors}

Many participants felt that if individual-level variables were to continue to be considered, the traditional examination of risk factors should give way to a greater consideration of protective factors. Several participants strongly emphasised that a much deeper understanding is needed of people's capacity for resilience, hope and 
optimism. They felt that if these constructs were better understood, society would be better equipped to prevent suicide. Suggestions included looking at different levels of resilience in different communities, and examining why individuals in similar circumstances respond to traumatic and challenging events with different levels of optimism. One participant put this succinctly, claiming:

'Research should be looking at what are the pathways to hope (and despair) in our society.'

\section{Methodological and related issues}

A number of participants commented on the overall direction of suicide prevention research to date, making suggestions about how different methodological approaches could improve the knowledge base.

Participants saw the value in real-world research, and advocated for conducting rigorous evaluations alongside funded service delivery projects in a way that could provide evidence of effectiveness. One participant summed up the strength of this approach in the following way:

'Operational and applied research is more important than that done under artificial conditions.'

A strong, recurring theme, however, was that deeper, more detailed and longer term research provided better quality and more useful information. Most felt that the research and evaluation efforts that sat alongside short term pilot projects were insufficient to contribute to evidence about what works and what does not work, and concluded that there should be 'no more pilots!' A number commented that it would be more useful to have fewer projects funded to a greater degree, with strong evaluation components built in. One participant who had just completed a rigorous evaluation as part of a tailored service delivery project noted that,

'if we had stopped at 12-18 months we would have had nothing, only ideas, not hard evidence.'

Several participants commented on the need to involve people in meaningful ways in any research into suicide prevention. This point was summed up in the following way by one participant:

'Participate with people so they understand what we are doing and why we are doing it with them.'

There was seen to be an educative element to this approach, in that it could empower those involved in the research to become effective community agents in the area of suicide prevention. This was seen to be especially important for particular groups, such as people in remote Indigenous communities with few resources.

Multidisciplinary research was also emphasised. This occurred in the context of several participants noting that suicide is not simply a medical problem, but also a social problem, and commenting that this therefore required the involvement of anthropologists and sociologists as part of research teams.

A few participants made mention of the fact that secondary analyses of existing data could complement the kind of primary research described above. Specific examples of routinelycollected datasets that could inform questions related to suicide prevention included coronial data and data from telephone help lines.

Several participants noted that irrespective of whether research used primary or secondary data sources, it needed to emphasise the Australian context. Several examples were cited where Australia-specific studies are required, including studies of community structure and interventions to improve social cohesion, studies of male behaviour (including reticence with communication and help-seeking) in the Australian culture, and studies of differing protective factors in multicultural Australia. Some participants advocated a large-scale longitudinal study that followed a large cohort from infancy to adulthood, examining risk and protective factors for suicide.

\section{Utilising research findings}

Many participants indicated that research findings are not used optimally by relevant stakeholders. Some felt that researchers could do more to translate their findings into practice. One, for example, observed that research not only required recommendations but also action statements and commitments by researchers to follow them up. Others noted that researchers could do more to disseminate their findings in meaningful ways to communities, policymakers, planners, clinicians and other practitioners, non-government representatives, other researchers, and those who use health and mental health services. 
On a related note, a number of participants talked about the need to raise the profile of suicide as a public health issue and suggested that the media has a role to play here. In part, this role involves disseminating research findings (e.g., about risk and protective factors and about interventions that show promise). The role may be broader than this, however, and could involve reducing stigma, changing culture and encouraging help-seeking behaviour. Participants acknowledged the need to conduct media campaigns and related activities with caution, given the strong evidence that reporting of suicide can lead to copycat behaviours. They suggested that media activities themselves would need to be closely evaluated.

\section{Developing a framework for suicide prevention that could guide suicide prevention research}

Several participants drew together the above themes in a call for a cohesive framework for suicide prevention that could guide suicide prevention research. A number felt that the approach to date had been somewhat ad hoc, and that more strategic directions were necessary, contrasting the current approach to suicide prevention with that used to tackle the road toll. One participant summarised the idea in the following way:

'We need a framework against which we place criteria - macro-level for the whole population, such as education at a young age about resilience and protective factors, and then at the micro-level, training about interventions that can help us identify risk factors.'

\section{Discussion and conclusions}

The current study was not without its limitations and some caution should be exercised in generalising the findings from the group interviews to other, broader groups of stakeholders. The purposeful sampling strategy and the relatively small total number of participants may have meant that the views of some major players were missed. In addition, although we explicitly invited potential stakeholders to participate based on their conducting, using and/or funding suicide prevention research and/or having been affected by suicide, and we know anecdotally that many stakeholders fell into more than one group, we did not systematically collect this information so we cannot profile our stakeholders. Having said this, our own knowledge of the Australian suicide prevention field gives us confidence that our sample was reasonably inclusive. In addition, the qualitative data from the group interviews reached the point of 'saturation', where no new ideas were being expressed (Patton, 1990), and the findings were consistent with those of the broader group of stakeholders who responded to the questionnaire in Study 1 (Robinson et al., 2008).

The above limitations aside, the current paper provides some guidance with respect to the direction Australia's suicide prevention research agenda could take. To summarise, group interview participants stressed that in terms of future suicide prevention research efforts, priority should be given to evaluating the efficacy of specific interventions and examining the response of the health and community service systems. They felt that the epidemiological profile of suicidal individuals had been explored, at least with respect to rates and individual-level risk factors, and that the above evaluative activities should focus on groups identified as having particularly high levels of risk. Most saw limited value in continuing to explore individual-level risk factors ad infinitum, and felt that the time had come to move on to considering wider societal influences on suicide and individual-level protective factors. Many felt that evaluation efforts should employ mixed methods, should be multidisciplinary and should be relevant to the Australian context. They also argued that there was scope for increasing the utility of research findings by communicating them in a manner that would enable them to be utilised by policy-makers, planners and practitioners. Several called for a more cohesive framework for suicide prevention that could guide suicide prevention research.

A number of these findings resonate with those of Study 1 in the current project, mentioned above (Robinson et al., 2008). Study 1 identified that, although granting bodies have provided some funding for intervention studies over the past seven years, this has only had a relatively small impact on the published literature. Instead, journal articles are dominated by epidemiological studies of rates, and, to a lesser extent, individual-level risk factors. Scant attention has been paid to individual-level protective factors, or to societal influences on suicide, and 
relatively little has been devoted to rigorous evaluations of interventions, services, policies and programs. It is understandable, therefore, that the demand for evaluative research from group interview participants was so strong; the questionnaire respondents in Study 1 also overwhelmingly gave priority to studies of interventions.

The predominance of epidemiological studies has meant that they have tended to rely on quantitative data collected by researchers with a biomedical focus. This has contributed to quantifying the magnitude of suicide as a problem in the Australian community, and has highlighted particular groups who are at high risk. Arguably, however, it has occurred at the expense of qualitative studies designed to explore the experiences of those who have been affected by suicide and those who provide services for them. Again, it is perhaps not surprising that group interview participants perceived this as an imbalance, and called for more qualitative, multidisciplinary studies of the trajectory of suicidal thoughts and behaviours, of why some people choose to seek help and others do not, and of what elements of particular interventions seem to be successful for particular individuals and groups.

The views of stakeholders are clearly an important information source that should be heeded in the process of setting research priorities. However, additional, more objective information is also required. In particular, data on the significance of the given problem should underpin priority-setting decisions. In the current study, for example, stakeholders expressed divergent views about the particular target groups that should be afforded research attention. Standard assessments of the relative burden of suicide for different groups could help to clarify where future efforts could be directed, and could be sought as part of the priority-setting process. As noted above, there is a considerable amount of epidemiological information available on group-specific rates of suicide, for example, and these data could be harnessed in Australia's current priority-setting exercise. It was beyond the scope of the current study to do this, but we would advocate such an objective examination of the epidemiological data. This is consistent with the international and Australian literature on priority-setting in public health research, which emphasises significance as a key criterion (Carson, Ansari \& Hart, 2000; World Health Organization, 1996).

In summary, the current paper indicates that in future Australia's suicide prevention research agenda should emphasise the evaluation of specific interventions, policies, programs and services. A priority-driven approach to suicide prevention research may ensure that the research endeavour provides the most useful information for those whose day-to-day work involves trying to prevent suicide.

\section{Note}

The Stolen Generation is a term used to describe the Aboriginal children, often of mixed descent, who were removed from their families by Australian government agencies and church missions prior to the 1970s.

\section{Acknowledgements}

This study was funded by the Australian Government Department of Health and Ageing. The authors would like to thank Professor Tony Jorm for conceptual advice.

\section{References}

Australian Government Department of Health and Ageing (2008a). Living Is For Everyone (LIFE) Framework: A Framework for Suicide Prevention in Australia. Canberra: Australian Government Department of Health and Ageing. (Available online http://www.livingisforeveryone.com.au/LIFEFramework.html)

Australian Government Department of Health and Ageing (2008b). Living Is For Everyone (LIFE) Framework: Research and Evidence in Suicide Prevention. Canberra: Australian Government Department of Health and Ageing. (Available online http://www.livingisforeveryone.com.au/Researchand-evidence-in-suicide-prevention.html)

Carson, N., Ansari, Z., \& Hart, W. (2000). Priority setting in public health and health services research. Australian Health Review, 23, 46-57.

Coreil, J. (1995). Group interview methods in community health research. Medical Anthropology, $16,193-210$.

Liamputtong Rice, P. \& Ezzy, D. (1999). Qualitative Research Methods. Melbourne: Oxford University Press.

Linehan, M.M. (2008). Suicide intervention research: A field in desperate need of development. Suicide and Life-Threatening Behavior, 38, 483-485. 
Mann, J.J., Apter, A., Bertolote, J., Beautrais, A., Currier, D., Haas, A. et al. (2005). Suicide prevention strategies: A systematic review. Journal of the American Medical Association, 294, 2064-2074.

Morse, J.M. \& Field, P.A. (1995). Qualitative Research Methods for Health Professionals (2nd edition). London: SAGE Publications.

Patton, M.Q. (1990). Qualitative Evaluation and Research Methods. Newbury Park, CA: Sage Publications.

Robinson, J., Pirkis, J., Krysinska, K., Niner, S., Jorm, A., Dudley, M., Schindeler, E., De Leo, D., \& Harrigan, S. (2008). Research priorities in suicide prevention: A comparison of current research efforts and stakeholder-identified priorities. Crisis, 29, 180190.

Streubert, H. \& Carpenter, D. (1999). Qualitative Research in Nursing (2nd edition). Philadelphia: JB Lippincott.

World Health Organization (1996). Investing in Health Research and Development: Report of the Ad Hoc Committee on Health Research Relating to Future Intervention Options. Geneva: World Health Organization. 